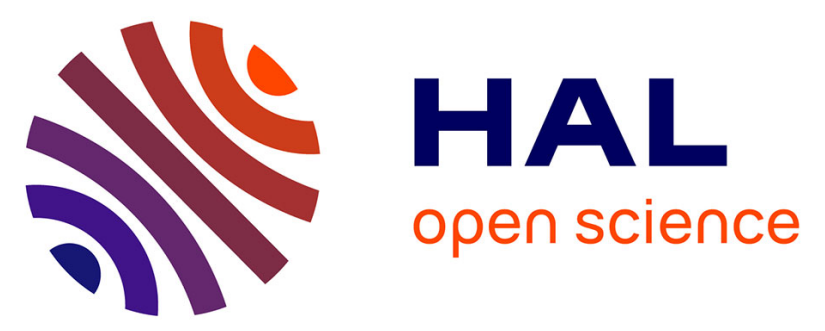

\title{
Evènements éoliens rapides dans les Loess du Pléniglaciaire supérieur Weichselien : l'exemple de la séquence de Nussloch (vallée du Rhin-Allemagne)
}

Pierre Antoine, Denis Didier Rousseau, Christine Hatté, Ludwig Zöller, Andréas Lang, Michel Fontugne, Olivier Moine

\section{To cite this version:}

Pierre Antoine, Denis Didier Rousseau, Christine Hatté, Ludwig Zöller, Andréas Lang, et al.. Evènements éoliens rapides dans les Loess du Pléniglaciaire supérieur Weichselien: l'exemple de la séquence de Nussloch (vallée du Rhin-Allemagne). Quaternaire, 2002, 13 (3), pp.199-208. 10.3406/quate.2002.1712 . hal-02497460

\author{
HAL Id: hal-02497460 \\ https://hal.science/hal-02497460
}

Submitted on 14 Oct 2020

HAL is a multi-disciplinary open access archive for the deposit and dissemination of scientific research documents, whether they are published or not. The documents may come from teaching and research institutions in France or abroad, or from public or private research centers.
L'archive ouverte pluridisciplinaire HAL, est destinée au dépôt et à la diffusion de documents scientifiques de niveau recherche, publiés ou non, émanant des établissements d'enseignement et de recherche français ou étrangers, des laboratoires publics ou privés. 
Evènements éoliens rapides dans les Loess du Pléniglaciaire supérieur Weichselien : l'exemple de la séquence de Nussloch (vallée du Rhin-Allemagne)

Pierre Antoine, Denis Didier Rousseau, Christine Hatté, Ludwig Zöller, Andréas Lang, Michel Fontugne, Olivier Moine

\section{Citer ce document / Cite this document :}

Antoine Pierre, Rousseau Denis Didier, Hatté Christine, Zöller Ludwig, Lang Andréas, Fontugne Michel, Moine Olivier. Evènements éoliens rapides dans les Loess du Pléniglaciaire supérieur Weichselien : l'exemple de la séquence de Nussloch (vallée du Rhin-Allemagne). In: Quaternaire, vol. 13, n³-4, 2002. Événements rapides, instabilités, changements culturels au Quaternaire. pp. 199-208;

doi : https://doi.org/10.3406/quate.2002.1712

https://www.persee.fr/doc/quate_1142-2904_2002_num_13_3_1712

Fichier pdf généré le 19/04/2018 


\title{
Résumé
}

L'étude des variations de la granulométrie et de la susceptibilité magnétique dans la séquence de Nussloch (Vallée du Rhin), où le taux de sédimentation est particulièrement élevé (1 à $2 \mathrm{~m} / \mathrm{ka}$ sur plus de $10 \mathrm{~m}$ ), débouche sur la mise en évidence de variations plus ou moins cycliques entre 30 et $17 \mathrm{ka}$ environ. Ces vanations, particulièrement bien soulignées par l'évolution du rapport \% 20-50um / \% $<20$ um (indice IGR), sont considérées comme une mesure indirecte des variations de la dynamique éolienne et du taux de sédimentation au moment du dépôt des matériaux loessiques (indice élevé dans les lœss typiques non altérés: "Lœss Events" L 1 à L 8 / indice faible dans les niveaux de gley de toundra GI à G8 (sédimentation réduite à nulle, développement du permafrost...). Sur la base des datations OSL et $14 \mathrm{C}$ sur loess on démontre qu'au cours du Pléniglaciaire supéneur l'accumulation loessique, particulièrement rapide et discontinue, correspond donc à une succession de phases de dépôt rapides séparées par des périodes d'arrêt ou de forte réduction du flux éolien de quelques siècles. Une tentative de corrélation de la courbe de I'IGR avec les vanations du taux de poussières dans les glaces du Groenland (GRIP) est proposée ; on observe par ailleurs qu'un certain parallélisme se dessine entre le niveau Henrich $\mathrm{H}-2$ et une des phases d'accumulation loessique les plus marquée située vers 22-23 ka. Enfin, la comparaison entre les variations de la susceptibilité magnétique et des données granulométriques montre que les périodes caractérisées par un indice granulométrique élevé se distinguent aussi par une augmentation de la proportion de minéraux ferromagnétiques remaniés à partir des zones de déflation de la plaine alluviale du Rhin en contrebas du site (augmentation de la fréquence et de l'intensité des tempêtes de N-NW).

\begin{abstract}
Variations of detailed grain size and magnetic susceptibility records in the Nussloch loess sequence (Rhine Valley), where the sedimentation rate is especially high ( 1 to $2 \mathrm{~m}$ per ka on more than $10 \mathrm{~m}$ ) are presented. This study leads to evidence more or less cyclic variations of these indices between about 30 and $17 \mathrm{ka}$. These variations, especially well expressed by the evolution of the IGR (\% 20-50um / \% $<20 \mathrm{um}$ ), are considered to be an indirect measurement of the aeohan dynamic intensity and of the sedimentation rate dunng loess deposition (high ratio in typical unweathered loess (Loess events $\mathrm{LI}$ to L8) / low ratio in tundra gleys horizons GI to G8 (reduction or stop of the sedimentation, permafrost development...). On the basis of OSL, and of I4C dates on loess organic matter, we show that dunng the Upper Pleniglacial the loess deposition was especially fast and discontinuous, and resulted from a succession of rapid deposition phases, separated by stops or strong reduction of the aeolian flux sedimentation dunng some centunes. A temptative correlation with the Greenland dust record (GRIP) is proposed. In addition we can observe a parallel between Henrich 2 event and one of the main period of loess deposition around 22-23 ka. Finally, the comparison with the magnetic susceptibility record and the grain-size data show that the period charactenzed by a high grain-size index, are also underlined by an increase in the amount of ferromagnetic minerals reworked from the Rhine alluvial plain located at the foot of the site (enhancement in the frequency of the storms from N-NW).
\end{abstract}

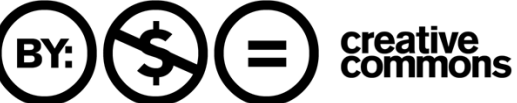




\title{
ÉVÉNEMENTS ÉOLIENS RAPIDES DANS LES LOESS DU PLÉNIGLACIAIRE SUPÉRIEUR WEICHSELIEN : L'EXEMPLE DE LA SÉQUENCE DE NUSSLOCH (VALLÉE DU RHIN-ALLEMAGNE)
}

\author{
Pierre ANTOINE', Denis-Didier ROUSSEAU ${ }^{2,3}$, Christine HATTÉ $^{4}$, \\ Ludwig ZÖLLER ${ }^{5}$, Andreas LANG ${ }^{6}$, Michel FONTUGNE ${ }^{7}$ et Olivier MOINE ${ }^{2}$
}

\begin{abstract}
RÉSUMÉ
L'étude des variations de la granulométrie et de la susceptibilité magnétique dans la séquence de Nussloch (Vallée du Rhin), où le taux de sédimentation est particulièrement élevé ( 1 à $2 \mathrm{~m} / \mathrm{ka}$ sur plus de $10 \mathrm{~m}$ ), débouche sur la mise en évidence de variations plus ou moins cycliques entre 30 et $17 \mathrm{ka}$ environ. Ces variations, particulièrement bien soulignées par l'évolution du rapport \% 20 $50 \mu \mathrm{m} / \%<20 \mu \mathrm{m}$ (indice IGR), sont considérées comme une mesure indirecte des variations de la dynamique éolienne et du taux de sédimentation au moment du dépôt des matériaux loessiques (indice élevé dans les lœess typiques non altérés : "Lœess Events" $\mathbf{L} 1$ à $L$ 8 / indice faible dans les niveaux de gley de toundra G1 à G8 (sédimentation réduite à nulle, développement du permafrost...). Sur la base des datations OSL et ${ }^{14} \mathrm{C}$ sur loess on démontre qu'au cours du Pléniglaciaire supérieur l'accumulation loessique, particulièrement rapide et discontinue, correspond donc à une succession de phases de dépôt rapides séparées par des périodes d'arrêt ou de forte réduction du flux éolien de quelques siècles. Une tentative de corrélation de la courbe de l'IGR avec les variations du taux de poussières dans les glaces du Groenland (GRIP) est proposée ; on observe par ailleurs qu'un certain parallélisme se dessine entre le niveau Henrich $\mathrm{H}-2$ et une des phases d'accumulation loessique les plus marquée située vers 22-23 ka. Enfin, la comparaison entre les variations de la susceptibilité magnétique et des données granulométriques montre que les périodes caractérisées par un indice granulométrique élevé se distinguent aussi par une augmentation de la proportion de minéraux ferromagnétiques remaniés à partir des zones de déflation de la plaine alluviale du Rhin en contrebas du site (augmentation de la fréquence et de l'intensité des tempêtes de N-NW).
\end{abstract}

Mots-clés : loess, granulométrie, événements rapides, Weichselien, Allemagne, Nussloch.

\section{ABSTRACT}

RAPID AEOLIAN EVENTS WITHIN THE WEICHSELIAN UPPER PLENIGLACIAL LOESS : THE EXAMPLE OF THE NUSSLOCH SEQUENCE (RHINE VALLEY, GERMANY).

Variations of detailed grain size and magnetic susceptibility records in the Nussloch loess sequence (Rhine Valley), where the sedimentation rate is especially high ( 1 to $2 \mathrm{~m}$ per ka on more than $10 \mathrm{~m}$ ) are presented. This study leads to evidence more or less cyclic variations of these indices between about 30 and $17 \mathrm{ka}$. These variations, especially well expressed by the evolution of the IGR $(\% 20-50 \mu \mathrm{m} / \%<20 \mu \mathrm{m})$, are considered to be an indirect measurement of the aeolian dynamic intensity and of the sedimentation rate during loess deposition (high ratio in typical unweathered loess (Loess events L1 to L8) / low ratio in tundra gleys horizons G1 to G8 (reduction or stop of the sedimentation, permafrost development...). On the basis of OSL, and of ${ }^{14} \mathrm{C}$ dates on loess organic matter, we show that during the Upper Pleniglacial the loess deposition was especially fast and discontinuous, and resulted from a succession of rapid deposition phases, separated by stops or strong reduction of the aeolian flux sedimentation during some centuries. A temptative correlation with the Greenland dust record (GRIP) is proposed. In addition we can observe a parallel between Henrich 2 event and one of the main period of loess deposition around 22-23 ka. Finally, the comparison with the magnetic susceptibility record and the grain-size data show that the period characterized by a high grain-size index, are also underlined by an increase in the amount of ferromagnetic minerals reworked from the Rhine alluvial plain located at the foot of the site (enhancement in the frequency of the storms from N-NW).

Key-words : Loess, grain-size, rapid events, Weichselian, Germany, Nussloch.

1 - Laboratoire de géographie physique - CNRS - I place A. Briand. 92195 Meudon Cedex - E-Mail : Pierre.Antoine@cnrs-bellerre.fr

2 - Université Montpellier II. Paléoenvironnements \& Palynologie. Institut des Sciences de l'Evolution (UMR CNRS 5554), case 61, pl. E. Bataillon, F-34095 Montpellier cedex 05

3 - Lamont-Doherty Earth Observatory of Columbia University, Palisades, NY 10964, USA - E-mail : denis@dstu.univ-montp2.fr

4- LSCE. CNRS-CEA, Avenue de la Terrasse, F-91198 Gif/Yvette Cedex. - E-mail : Christine.Hatte@ lsce.cnrs-gif.fr / Michel.Fontugne@lsce.cnrs-gif.fr

5 - Lehrstuhl Geomorphologie. Universitaet Bayreuth.95440 Bayreuth. Germany, - E-mail : ludwig.zoeller@uni-bayreuth.de

6 - Fysische en Regionale Geografie, K.U. Leuven Redingenstraat 16. 3000 Louvain, Belgique, - E-mail: Andreas.Lang@geo.kuleuven.ac.be 


\section{I - INTRODUCTION}

La coupe de Nussloch (Fig. 1), décrite en premier lieu par Sabelberg et Lösher (1978), Bente et Lösher (1987), et datée par Zöller et al., (1988), Zöller et Wagner (1990) puis Zöller et Lang (in Antoine et al., 2001; Hatté et al., 1998, 1999), a été choisie pour ces études en raison du développement exceptionnel des loess weichseliens qui la caractérise $(18 \mathrm{~m})$.

L'étude pluridisciplinaire des différents profils de loess de Nussloch, qui a débuté en 1985 dans le cade du Programme Européen BIMACEL (Biological and Magnetic Records and dating of climatic Changes in Western European Loess series), est actuellement soutenue par un financement du CNRS (Programme ECLIP$\mathrm{SE}$ ). Les données stratigraphiques, sédimentologiques et géochronologiques détaillées, ainsi que les premiers résultats de l'analyse du $\delta^{13} \mathrm{C}$ ont été publiés récemment et ne seront pas exposés dans ce court article (Antoine $e t$ al., 2001, Hatté et al., 1998, 1999, 2001).

Le contexte géomorphologique de ce secteur du flanc est de la haute vallée du Rhin (Upper Rhine Graben) se caractérise par la présence d'une très large plaine alluviale ( $30 \mathrm{~km}$ env.), séparée du plateau de l'Odenwald par un versant abrupt exposé à l'W-NW (Fig. 1). Au cours du Weichselien, cette morphologie particulière a favorisé l'accumulation d'épaisses séries loessiques à la jonction entre le plateau de l'Odenwald et le versant de la rive droite du Rhin. Ce phénomène a produit une succession de rides de loess allongées de 15 à $20 \mathrm{~m}$ d'épaisseur sur 2 à $4 \mathrm{~km}$ de longueur orientées N-NW / S-SE, séparées par des vallons sccs (Fig. 1). Cette morphologie spécifique correspond à celle des "gredas" observées en Europe centrale, où elles s'orientent parallèlement à la direction des vents responsables de la déflation et des dépôts loessiques (Léger, 1990). À Nussloch, cette accumulation est en grande partie liée à la présence d'une très large plaine alluviale parcourue par un système de chenaux en tresses au cours du dernier maximum glaciaire (Bibus, 1980), qui représentait alors une abondante source de sables et de limons pour la déflation éolienne. Enfin, en raison de sa situation géomorphologique, la séquence de Nussloch se distingue par des processus sédimentaires essentiellement dominés par une dynamique éolienne et comporte peu de dépôts remaniés par ruissellement et (ou) colluvionnement. Ces caractéristiques en font un des meilleurs profils pour l'étude des loess weichseliens de l'Europe du Nord-Ouest et plus particulièrement pour ceux du Pléniglaciaire supérieur qui y sont représentés par 10 à $14 \mathrm{~m}$ de dépôts.

\section{II - DONNÉES STRATIGRAPHIQUES}

La stratigraphie des loess du Pléistocène supérieur de Nussloch est basée sur l'étude pédostratigraphique détaillée et la corrélation de 4 profils (Fig. 2). Elle débouche sur la mise en évidence d'une séquence complexe comportant 4 grands ensembles stratigraphiques principaux (Fig. 2) (seuls les profils $\mathrm{P} 2$ à $\mathrm{P} 4$, où le Pléniglaciaire supérieur est le plus dilaté, sont repris ici).

\section{Eemien et Début-Glaciaire weichselien (120-75 ka env.)}

Le complexe de sols basal de Nussloch est essentiellement représenté par un horizon $\mathrm{Bt}$ complexe de sol brun lessivé tronqué $(2 \mathrm{a} / 2 \mathrm{~b})$, développé sur un loess calcaire (unité 1 , Saalien). Ce sol, attribué principalement à l'interglaciaire Eemien, est recouvert par des colluvions argileuses qui enregistrent la dernière pédogenèse de type gris-forestier du Début-Glaciaire (unités $3 \& 4$, Sous-stade 5a), puis par un sol isohumique steppique

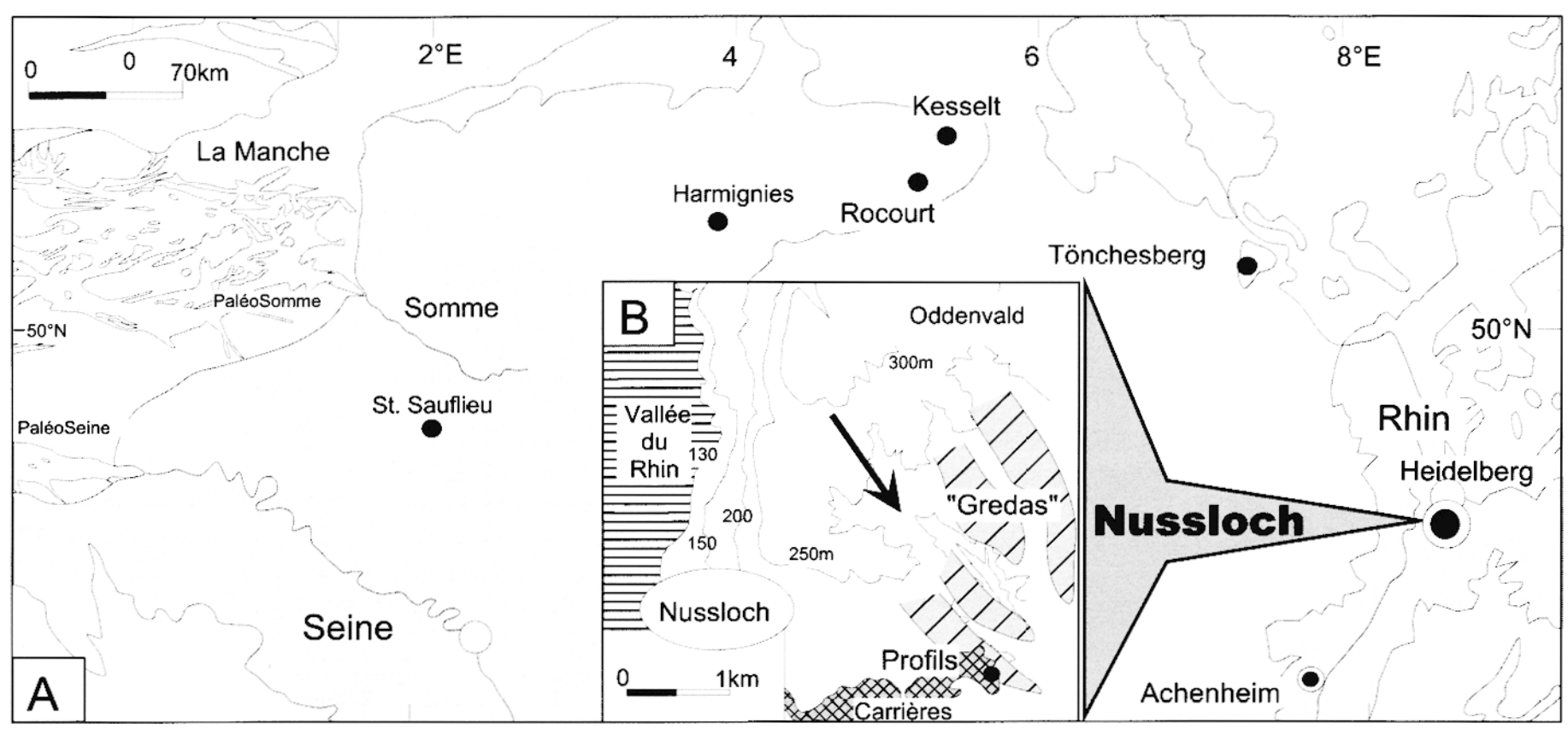

Fig. 1 - Localisation de la séquence de Nussloch dans la zone loessique de l'Europe du Nord-Ouest. Fig. 1 - Location of the Nussloch sequence within the northwestern European loess belt. 
développé sur dépôts éoliens à la limite des stades isotopiques $5 \mathrm{a}$ et 4 (unité 5 - Mosbacher Humuszone)

\section{Pléniglaciaire inférieur (75-55ka env.) :}

L'ensemble II se distingue par des loess calcaires grisâtres à horizons hydromorphes (unités 9 à 12 et P4-1, indifférenciées dans la figure 2), et se termine par la mise en place de sables et de limons éoliens qui prennent localement la forme d'une dune de 5 à $6 \mathrm{~m}$ d'épaisseur entre P1 et P2 (unité 13).

\section{Pléniglaciaire moyen (55-30ka env.)}

Cette partie de la séquence se distingue nettement des ensembles sous et sus-jacents par l'importance relative des faciès d'altération : sols bruns $\mathrm{n}^{\circ} \mathrm{P} 4-2,14$ et 20 (Löhner Böden), et le faible développement de la sédimentation loessique (2-4m max).

\section{Pléniglaciaire supérieur (15-30ka)}

Le Pléniglaciaire supérieur se distingue nettement par un énorme développement des loess calcaires typiques $(10$ à $14 \mathrm{~m})$ qui débute au sommet du sol brun 20 "Löhner böden" comme dans la plupart des autres séquences loessiques de la vallée du Rhin (Frechen, 1999). D'après les données granulométriques moyennées ( 100 éch. $/ 10 \mathrm{~m})$, ces loess s'individualisent par un fort pourcentage de sables fins $(50-200 \mu \mathrm{m}: 7,9 \%)$, de faibles teneurs en argile $(<2 \mu \mathrm{m}: 9,2 \%)$, et un fort pourcentage de carbonates $(23,8 \%)$. Au sein de cette séquence on peut distinguer trois faciès principaux de la base au sommet :

- Une unité basale de loess calcaire homogène (unité 22),

- Un ensemble médian de loess calcaires lités à microfentes de cryo-dessication (unités 24 à 34) à gleys de toundra cryoturbés dans la partie inférieure (unités 23, 25-26, 28,30,33 et $35:$ Nasboden). Ces loess constitués par la superposition de micro-séquences granodécroissantes de quelques millimètres à $1 \mathrm{~cm}$ d'épaisseur, séparées par les niveaux de fines fentes, présentent des caractéristiques proches de celles des loess lités nivéoéoliens du Nord de la France et de la Belgique (Lautridou et Sommé, 1974, Haesaerts, 1985).

Cette partie de la séquence a enregistré une retombée volcanique (Fig. 3 : E.T. épaisseur 2 à $5 \mathrm{~mm}$ ), toujours observée au sein de la dernière unité de loess lité (34) où elle est déformée par des petites fentes de cryodessication. Ce niveau repère est corrélé avec l'Eltviller tuf (Juvigné et Semmel, 1981), daté de 19 à $20 \mathrm{ka}$ d'après les nouveaux résultats OSL obtenus à Nussloch (Antoine et al., 2001).

- Deux unités supérieures de loess calcaires, homogènes plus grisâtre (36 et 39) incluant un petit gley non perturbé, séparées des loess lités par un horizon grisâtre à fentes déformées (35). D'après sa position stratigraphique et les datations $\mathrm{OSL}$ et ${ }^{14} \mathrm{C}$, cet horizon grisâtre pourrait être corrélé avec l'horizon à langues de Nagelbeek (Haesaerts et al., 1981).

Les gleys de toundra qui ponctuent cette séquence surtout dans sa partie médiane (Nassboden Erbenheim E1 à E4/E5 des auteurs allemands), se traduisent par une hydromorphie (coloration gris bleuté), une légère décar- bonatation avec redistribution des carbonates en bas du profil (poupées), la réduction du fer (plages et linéoles oxydées) et un léger enrichissement en carbone organique (enracinement et activité biologique plus importante). En ce qui concerne les faunes malacologiques, la proportion des espèces hygrophiles augmente dans les gleys, contrairement à celle des espèces de terrain découvert sec et ensoleillé, et mésophiles, qui ont plutôt tendance à diminuer. Toutefois, ces baisses de proportion sont moins prononcées chez les espèces mésophiles beaucoup moins inféodées aux habitats très secs (Moine et al., ce volume). Ces gleys de toundra traduisent de courtes périodes d'arrêt ou de forte réduction de la sédimentation loessique dans un environnement très froid et relativement plus humide avec développement de permafrost (cryo-injections) et localement de fentes à coin de glace (ice wedge), comme dans d'autres séquences loessiques européennes (Haesaerts et Van Vliet-Lanoë, 1981, Van Vliet-Lanoë, 1987, Vandenberghe et al., 1998). On remarque d'ailleurs que dans la séquence du Pléniglaciaire moyen à sols bruns, des gleys similaires se développent systématiquement au sommet des sols interstadiaires (14 et 20) où ils signent à chaque fois une dégradation climatique précédant l'arrivée des loess.

La formation des gleys de toundra ne correspond donc à aucune amélioration climatique et n'est donc pas à mettre en relation avec des interstades. Cependant les données récentes issues de l'étude malacologique montrent néanmoins un net développement de la diversité des associations malacologiques au sommet des gleys de toundra, et parfois de brusques et importantes augmentations de l'abondance (Moine et al., ce volume). Cette observation pourrait traduire l'existence de courtes périodes d'amélioration climatique relatives entre le sommet des gleys et la base d'une nouvelle phase de dépôts loessique. Ces améliorations climatiques rapides, non détectables dans la stratigraphie (hiatus sédimentaire), pourraient par ailleurs être à l'origine des épisodes de dégradation du permafrost qui marquent le sommet des gleys de toundra (fonte des coins de glace et fluage de la couche active comme dans la coupe d'Harmignies en Belgique (Haesaerts et Van Vliet-Lanoë, 1981).

D'une manière générale, l'étude des loess du Pléniglaciaire supérieur de Nussloch permet de mettre en évidence deux courtes phases très arides caractérisées par des loess homogènes (unités 22,36 et 38 ) encadrant une période plus humide et plus longue où la couverture nivale était plus développée entre environs 26 et $20 \mathrm{ka}$ (unités 24 à 34). Le caractère plus humide de cette période du début du Pléniglaciaire supérieur déjà relevé par plusieurs auteurs (Haesaerts, 1985 ; Huijzer and Vandenberghe, 1998), est en accord avec des précipitations plus abondantes responsables du développement maximal de l'inlandsis scandinave (Elverøi et al., 1995). Par contre une aridité maximale est notée dans les loess homogènes supérieurs par l'étude $d u \delta^{13} \mathrm{C}$ du carbone organique (Hatté et al., 1998), et plus particulièrement au niveau de l'Eltviller tuf vers 19-20ka BP. Compte tenu des datations, la phase médiane du Pléniglaciaire supérieur, au cours de laquelle se dépose l'essentiel des 


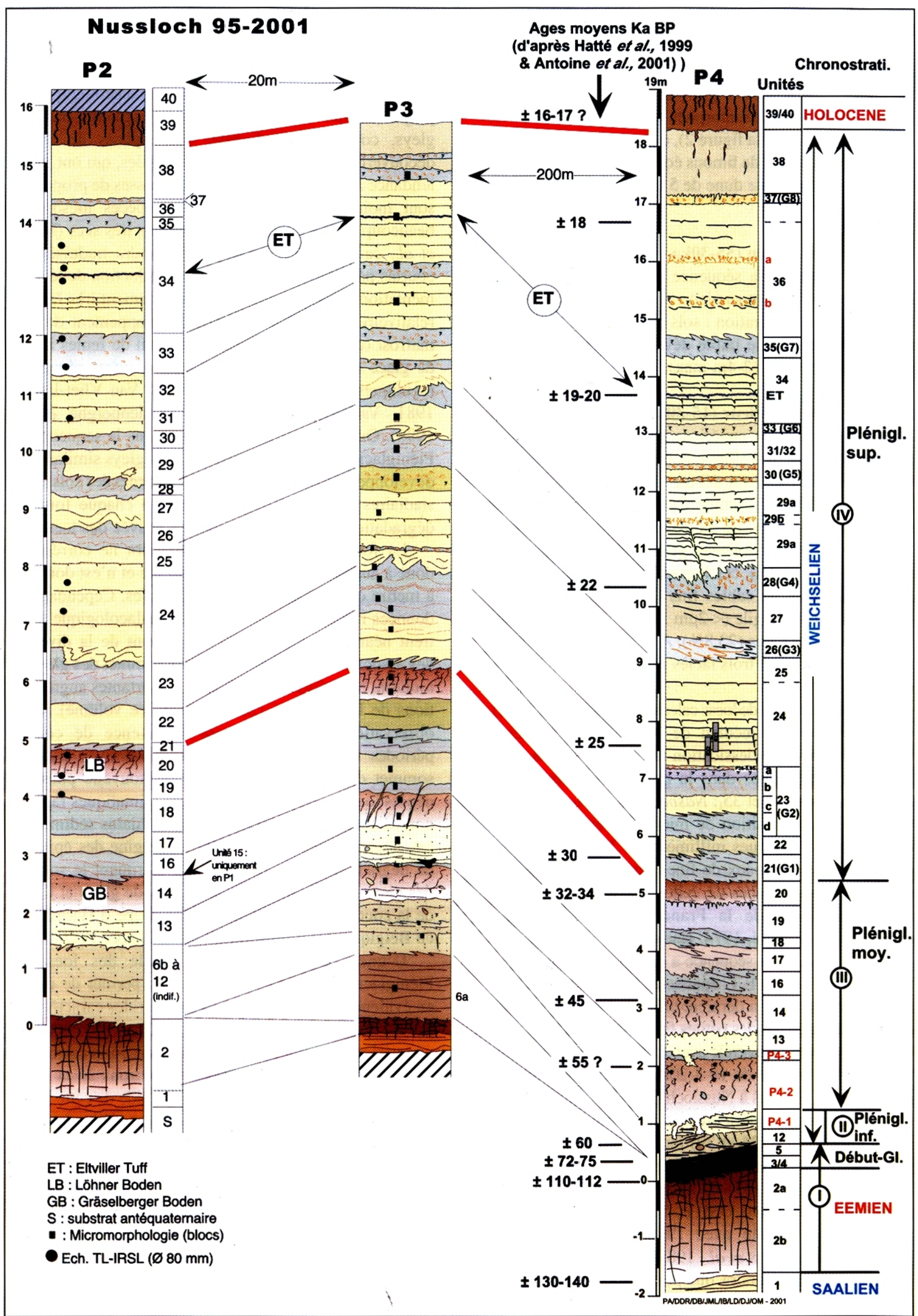


loess, est contemporaine des principaux pics de poussière enregistrés dans la glace du Groenland à GRIP entre 25 et 20ka BP (GRIP Members, 1993). Enfin, l'énorme développement des taux de sédimentation loessique (> $1000 \mathrm{~mm} / \mathrm{ka}$ ) au cours du Stade Isotopique 2 correspond à un phénomène généralisé dans les séquences d'Europe (Frechen, 1999 ; Antoine et al., 1998 ; Haesaerts et al., 1996a,b).

\section{III - VARIATIONS DE LA DYNAMIQUE ÉOLIENNE AU PLÉNIGLACIAIRE SUPÉRIEUR}

\section{IIIa -Variations de la susceptibilité magnétique}

Dans la plupart des séquences loessiques et plus particulièrement en Chine, il est actuellement admis que les valeurs les plus élevées de susceptibilité magnétique sont essentiellement liées à la néoformation de minéraux ferromagnétiques (principalement magnétite et maghemite) lors des pédogenèses (Zhou et al., 1990 ; Maher and Thompson, 1992 ; Heller and Evans, 1995). Ce phénomène est relié à l'activité bactérienne qui se développe dans les sols (magnetotactic bacteria, Maher and Taylor, 1988). Des travaux récents menés dans le Nord de la France ont par ailleurs montré que les valeurs maximales étaient liées aux sols humifêres de type gris-forestiers et aux sols isohumiques steppiques du Début-Glaciaire weichselien (Antoine et al., 1999), alors que les valeurs minimales caractérisent systématiquement les loess typiques allochtones (Rousseau et al., 1994, 1998).

Par contre dans les profils de Nussloch, excepté dans le complexe basal Eemien / Début-Glaciaire et surtout dans le sol postglaciaire, les variations de la susceptibilité magnétique apparaissent inversées par rapport aux variations classiques à commande pédologique (Fig. 3).

Les valeurs de susceptibilité les plus élevées apparaissent systématiquement dans les loess et plus particulièrement dans les loess du Pléniglaciaire supérieur (Fig. 3). Des minima sont par contre observés au niveau des gleys de toundra les plus développés (Fig. 3, G1 à G8). Ce phénomène est pour partie à mettre en relation avec l'altération des minéraux magnétique (transformation de la magnétite en hématite) caractéristique de ces niveaux hydromorphes (Nawrocki et al., 1996).

Compte tenu de ces observations et des données sédi- mentologiques précédemment exposées, ont peut penser que les valeurs élevées de susceptibilité magnétique relevées dans les loess de Nussloch (8 à 10 unités SI), par comparaison avec celles obtenues à Achenheim ou à St. Pierre-les-Elbeuf (Rousseau et al., 1994, Antoine et al., 1999), résultent d'un enrichissement en minéraux ferromagnétiques d'origine détritique autochtone (lithogenic origin). Des processus semblables débouchant sur des variations inverses de la susceptibilité magnétique ont d'ailleurs été décrits à Kurtak en Sibérie (Chlachula et al., 1998), en Alaska (Begét et al., 1990), et en Pologne (Nawrocki et al., 1996).

Cette interprétation concernant l'origine de la susceptibilité magnétique à Nussloch est confortée par la comparaison avec les données granulométriques des loess du Pléniglaciaire supérieur. En effet, on remarque un parallélisme assez net entre l'évolution de l'IGR (Fig. 3 ), et les variations de la susceptibilité magnétique dans ces loess. Cette observation tend à montrer que la forte susceptibilité des loess de Nussloch est liée à la présence de magnétite détritique dans la fraction $20-50 \mu \mathrm{m}$ (magnétite grossière).

Par contre les particules silteuses allochtones originaires des grandes zones de déflation de la Manche et de la Mer du Nord (Lautridou, 1985) et qui constituent le materiau originel principal des loess du nord-ouest européen sont caractérisées par de très faibles valeurs de susceptibilité (Rousseau et al. 1998 ; Antoine et al., 1998), correspondant à un faible contenu en magnétite.

D'après le contexte géomorphologique du site, exposé en introduction, l'origine des minéraux ferromagnétiques responsable de la susceptibilité élevée des loess de Nussloch est à rechercher dans les sédiments fluviatiles de la vallée du Rhin largement exposés à la déflation au cours du Pléniglaciaire supérieur. Une partie non négligeable du matériel limoneux constituant les loess de Nussloch est donc d'origine locale et son importance relative varie en fonction de la dynamique éolienne.

Cette relation entre la sédimentation éolienne à Nussloch et la déflation dans la plaine du Rhin est par ailleurs clairement établie par la présence de sables grossiers et de petits graviers ( 4 à $5 \mathrm{~mm}$ ), provenant de la vallée dans l'unité sableuse 13 du Pléniglaciaire inférieur (Fig. 2) et de lits de sable fin dans les loess lités du

Fig. 2 - Stratigraphie détaillée des profills P2, P3 et P4 de Nussloch

1- Loess calcaire (Saalien), 2a-2b - Sol brun lessivé tronqué (Eemien), 3, 4, 5 (Début-Glaciaire) Sol gris forestier sur colluvions

(3/4) 5 - Sol isohumique steppique, 6a-Colluvions sablo-limoneuses litées, (Unités 7 à 12 non représentées ici : uniquement présentes dans le profil P1, c.f. Antoine et al., 2001), 13 - Sables éoliens, P4-2, 14 et 20 : Sols bruns de type boréal (Cambisols)

(Pléniglaciaire moyen), 16 et 18: Gleys de toundra, 17 et $19:$ loess calcaires homogènes (Pléniglaciaire moyen),

21 à 38 : Séquence loessique du Pléniglaciaire supérieur: loess homogènes $(22,23 \mathrm{c}, 36,38)$, gleys de toundra plus ou moins cryoturbés $(21,23 a$,b,d, $26,28,30,33,35,37)$, loess lités à microfentes $(24,27,29 \mathrm{a}, 31,32,34), 39-40$ : Sol de surface post glaciaire.

Fig. 2 - Detailed stratigraphy of the loess section P2, P3 and P4 of Nussloch.

1- Calcareous loess (Saalian), 2a-2b-Truncated brown leached soil (Eemian), 3, 4, 5 (Early-Glacial) Grey forested soil on colluvium (3/4), 5 - Humic steppe soil, 6a-Laminated sandy-loamy colluvium. (Units 7 to 12 undiscribed hear: only observed in profile P1, see Antoine et al., 2001), 13- Aeolian sands P4-2, 14 and 20 : Boreal brown soil horizons (Cumbisols) (Middle Pleniglacial),

16 and 18: Tundra gleys (Middle Pleniglacial), 17 and 19 (homogeneous calcareous loess (Middle Pleniglacial),

21 to 38 : Upper Pleniglacial loess sequence: homogeneous loess $(22,23 \mathrm{c}, 36,38)$, more or less cryoturbated tundra gley horizons $(21,23 a, b, d, 26,28,30,33,35,37)$, laminated loess with cryodessication micnocracks $(24,27,29 a, 31,32,34)$, 39-40: surface soil (Postglacial). 


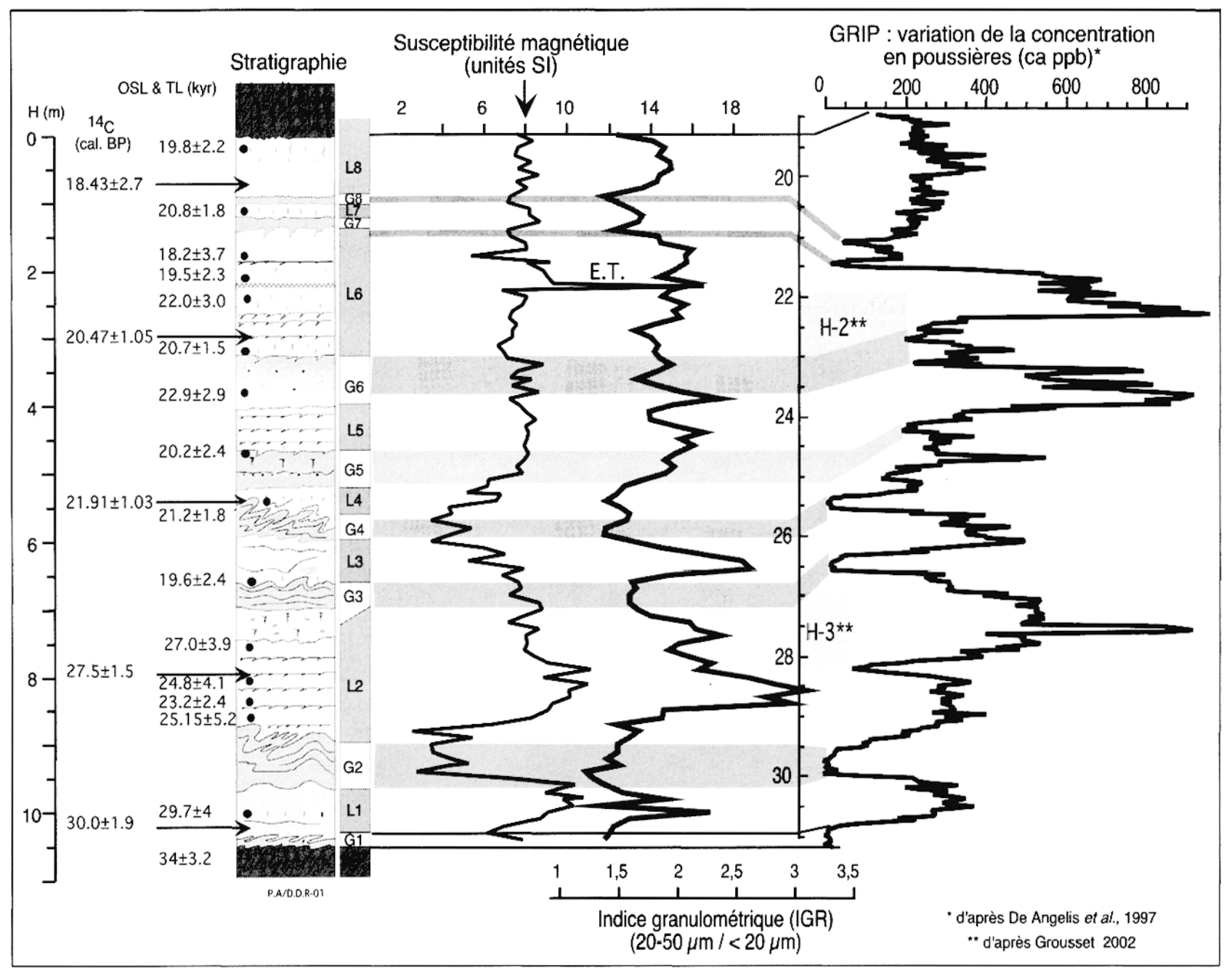

Fig. 3 - Essai de comparaison entre les variations de l'indice IGR dans les loess du Pléniglaciaire supérieur de Nussloch (d'après Antoine et al., 2001) et les variations du taux de poussière dans la glace à GRIP entre 31 et 19 ka (d'après De Angelis et al., 1997).

Fig. 3 - Attemp in comparison between the evolution of the grain size index (IGR)(Antoine et al., 200I) along the Upper Pleniglacial loess sequence at Nussloch and the variations of the dust content in the ice at GRIP between 31 and $19 \mathrm{ka}$ (according to De Angelis et al., 1997).

Pléniglaciaire supérieur. Compte tenu de la topographie et la position du site par rapport à la source de sable, les vents dominants responsables de la déflation et du transport des particules devaient venir du N-NW, parallèlement au grand axe des rides de loess (Fig. 1). Ces processus de remaniement éolien dans la vallée du Rhin sont aussi démontrés par l'existence de grandes dunes datées du Dryas récent en contrebas de Nussloch (Sandhausen). Le faciès de ces sables éoliens est d'ailleurs très proches de celui des sables de la dune de l'unité 13 et des lits sableux intercalés dans les loess lités du Pléniglaciaire supérieur.

Dans les profils de Nussloch, la susceptibilité magnétique associée à la granulométrie, peut donc être utilisées pour caractériser les variations de l'importance relative du remaniement de matériel local et donc l'importance de la dynamique éolienne. En effet, comme l'ont propo- sé Bégét et al., (1990) pour les loess d'Alaska, l'accélération des vents produit une augmentation de la proportion de magnétite dans les loess proches de la zone source.

\section{IIIb- Données granulométriques}

Au sein des loess du Pléniglaciaire supérieur l'étude de l'évolution en continu ( 1 éch. $/ 10 \mathrm{~cm}$ ) des différentes fractions granulométriques montre des variations rapides. Par exemple le pourcentage de limon grossier (LG: 20-50 $\mu \mathrm{m}$ ), caractéristique des loess, varie entre 55 et $67 \%$ dans l'ensemble de la séquence IV. Les variations du rapport $\% 20-50 \mu \mathrm{m} / \%<20 \mu \mathrm{m}$ (IGR), permettent de définir une succession de 3 à 4 grandes séquences de 1,4 à $2,5 \mathrm{~m}$ (Fig. 3). D'après les âges $\mathrm{OSL}$ et ${ }^{14} \mathrm{C}$, ces séquences pourraient représenter des périodes de 1 à 2,5 ka au cours desquelles les taux de sédimentation sont 
donc très élevés. On remarque que les valeurs maximales de la fraction $20-50 \mu \mathrm{m}$ correspondent généralement à des loess purs alors que les valeurs les plus faibles sont surtout marquées au niveau des gleys de toundra. Si l'on admet que la composition granulométrique des loess est un indicateur de la vitesse de transport des particules silteuses, il est possible de relier ces variations granulométriques à des variations de la vitesse des vents (Porter and An, 1995 ; Xiao et al., 1995 ; Vandenberghe et al., 1997). Les gleys de toundra correspondent donc à des périodes de diminution importante de l'apport loessique et de la vitesse des vents comme dans cela a été montré dans la séquence de Kesselt en Belgique (Vandenberghe et al., 1998).

Le taux de sédimentation moyen pour les $10 \mathrm{~m}$ supérieurs varie entre 1 et $2,5 \mathrm{~mm}$ par an compte tenu des deux bornes des intervalles de validité des dates OSL mais peu atteindre $4 \mathrm{~mm}$ dans les derniers $6,5 \mathrm{~m}$ de la séquence. Ces vitesses apparemment très élevées sont néanmoins inférieures à celles enregistrées lors des tempêtes de poussière actuelles dans la province chinoise de Gansu (Derbyshire et al., 1988 ).

Enfin, on remarque que les taux de sédimentation proposés sont globalement cohérents avec l'épaisseur des microséquences observées dans les loess lités de la séquence IV (unités 24 à 34). En effet l'épaisseur de ces séquences à granoclassement décroissant souvent soulignées par un lit sableux à la base, le plus souvent de l'ordre de 2 à $5 \mathrm{~mm}$, peut atteindre $1 \mathrm{~cm}$. Cette interprétation est renforcée par le regroupement des âges OSL dans les 6,5 m supérieurs de la coupe (20-21 ka BP) qui indique aussi un taux de sédimentation exceptionnellement élevé lors du Dernier Maximum Glaciaire.

D'une manière générale, ces variations granulométriques sont donc attribuées à l'alternance de périodes dominées par des tempêtes de poussière caractérisées par une granulométrie plus grossière et une augmentation de la part relative de l'alimentation locale (limons grossiers et sables), et d'épisodes plus calmes où le matériel limoneux plus fin d'origine allochtone domine. Le parallélisme déjà évoqué entre les variations de la susceptibilité magnétique et celles de la fraction 20$50 \mu \mathrm{m}$ des loess dans les $10 \mathrm{~m}$ supérieurs est en accord avec une accentuation du remaniement de matériel local provenant de la vallée du Rhin lors des épisodes éoliens les plus intensifs (Antoine et al., 2001).

$D$ 'après les données géochronologiques ces épisodes d'accentuation très brefs de la dynamique éolienne au cours du Pléniglaciaire supérieur sont globalement contemporains des pics de poussière enregistrés dans les glaces au même moment (Fig. 3). En effet, compte tenu des datations disponibles, une corrélation globale se dessine assez nettement entre les variations de l'indice IGR et celle des taux de poussières dans la glace à GRIP entre 21-22 et 28-29 ka (Fig. 3). En se basant sur la logique de la succession des phases de dépôt loessique (L1 à L8) et des gleys de toundra (G1 à G8) et les datations, il est même possible de proposer une corrélation plus fine entre les phases d'accumulation loessiques principales à IGR élevé, avec les pics majeurs de poussière de GRIP

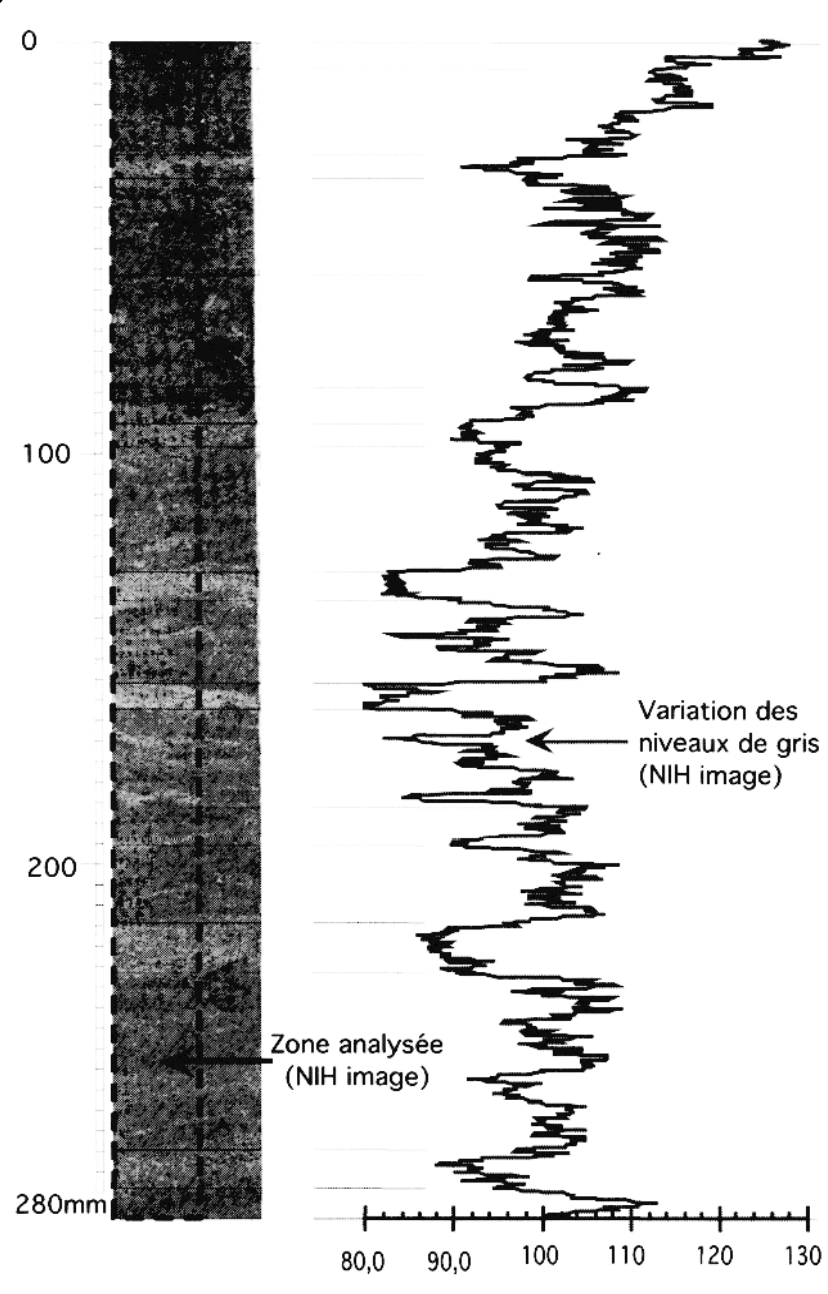

Fig. 4 - Analyse en niveau de gris d'un échantillon-barre $(280 \mathrm{~mm})$ de loess lité du Pléniglaciaire supérieur (unité 24), a l'aide du logiciel NIH Image.

Fig. 4 - Grey level analysis of a bar-sample $(280 \mathrm{~mm})$ from the Upper Pleniglacial loess (unit 24), using the NIH Image software.

et notamment avec ceux centrés vers $21,5-22,5,23,5$ 24,5 et 26,5-28ka (Fig. 3) (Rousseau et al., 2002). Ces travaux tendent donc à démontrer qu'il existe un lien direct entre les variations de la dynamique éolienne en Europe de l'Ouest et l'intensité de la circulation atmosphérique au niveau de l'Atlantique Nord. En effet, il est probable que l'augmentation de la fréquence et de l'intensité des dépressions atlantiques génére une accentuation de la déflation et du transport à haute altitude des particules limoneuses à partir des zones de déflation de la Manche Est et de la Mer du Nord (vents de NW à N$\mathrm{NW}$ ), parallèlement à l'intensification de la déflation dans les vallées de l'Europe du NW (augmentation des apports locaux en sable et en limon grossier).

Enfin, dans la partie finement litée de la séquence loessique de P4 (unité 24, Fig. 2) l'observation de terrain montre que ce faciès correspond à la succession de lits millimétriques à centimétriques montrant des forts contrastes granulométriques (alternances de niveaux plus 
ou moins riches en sable dérivés de la vallée du Rhin). Des échantillons ont été prélevés récemment afin de caractériser d'une manière plus fine la structure de ces dépôts (rails aluminium en $U$ de $50 \times 5 \times 3 \mathrm{~cm}$ ). Une tentative pour étudier leur structure fine a été développée sur la base d'images obtenues par numérisation directe des échantillons à une résolution de 300DPI avec un scanner de bureau (Fig. 4). L'image ainsi obtenue est ensuite importée dans le logiciel NIH Image (Porter, 2000), qui permet d'obtenir rapidement une courbe de variation des niveaux de gris intégrant l'intégralité de la surface de l'échantillon (Fig. 4).

Ces résultats permettent de mettre en évidence des variations à très haute résolution de la granulométrie de la colonne de loess. Dans cette partie de la séquence, l'accumulation loessique très rapide résulte clairement d'épisodes sédimentaires brutaux et discontinus de quelques millimètres à $1 \mathrm{~cm}$ d'épaisseur. On remarque par ailleurs que les épisodes les plus grossiers (niveaux sableux en gris clair dans la Fig. 4), qui traduisent des événements éoliens uniques particulièrement intenses (apports de la vallée du Rhin), correspondent aussi aux lits les plus épais $( \pm 1 \mathrm{~cm})$. Cette approche originale de la sédimentologie des loess nous permet donc de mieux comprendre les valeurs extrêmes des taux de sédimentation préalablement suggérés, notamment à partir des datations IRSL et ${ }^{14} \mathrm{C}$ (Antoine et al., 2001).

En effet, sur la base de ces observations, les taux de sédimentation peuvent localement atteindre 1 à $2 \mathrm{~cm}$ pour 10 ans dans ce type de dépôt, ce qui ouvre des perspectives intéressantes pour l'étude des variations de la dynamique éolienne à très haute résolution (variations séculaires). Afin de tester cette technique prometteuse sur un pas de temps plus important, un échantillonnage en continu du profil P4 de Nussloch a été effectué dans le but d'étudier la structure fine et de tester l'éventuelle cyclicité et périodicité de ces variations sur la période $20-30$ ka BP ( \pm 14 m d'épaisseur).

\section{IV - CONCLUSIONS}

L'étude à haute résolution de la variation des paramètres granulométriques et de la susceptibilité magnétique couplée avec une campagne de datation systématique dans la séquence de Nussloch débouche sur les conclusions suivantes :

1 - Contrairement à ce qui est observé dans la majeure partie des coupes européennes au cours du dernier cycle climatique, les variations de la susceptibilité à
Nussloch ne sont pas contrôlées par des phénomènes pédologiques, mais principalement par des variations dans la quantité de magnétite détritique incorporée aux loess. Au niveau des gleys de toundra le signal est cependant affecté par l'altération de la magnétite en contexte hydromorphe.

2 - Les variations granulométriques et notamment celles de la fraction 20-50 $\mu \mathrm{m}$ et du rapport $\% 20-50 \mu \mathrm{m} /$ $\%<20 \mu \mathrm{m}$ (IGR) permettent de définir une succession de séquences interprétées comme des cycles d'augmentation puis de réduction de l'intensité moyenne des vents. D'après les données ${ }^{14} \mathrm{C}$ et OSL ces séquences couvrent des périodes de quelques millénaires voire de quelques siècles pour les plus courtes. Au cours de ces périodes les taux de sédimentation sont extrêmement importants $(>1$ ou $2 \mathrm{~m} / \mathrm{ka}$ ) et semblent montrer une cyclicité d'ordre millénaire. Par ailleurs, une corrélation se dessine entre les pics de poussière principaux de GRIP et les phases majeures de dépôt loessique à IGR élevés. Ces données confirment le caractère fondamentalement discontinu de l'enregistrement loessique en Europe de l'Ouest et témoignent des relations qui existent entre la sédimentation loessique en Europe du NW et les variations du taux de poussière dans l'atmosphère. Néanmoins des analyses complémentaires, notamment la détermination des cortèges de minéraux lourds, sont prévues afin de tester les hypothèses concernant les relations entre les zones de déflation et de dépôts des matériaux loessiques.

3 - Le parallélisme entre les variations de la susceptibilité magnétique et de la fraction $20-50 \mu \mathrm{m}$, montre qu'au cours du Pléniglaciaire supérieur la sédimentation loessique allochtone a été localement perturbée par une alimentation locale caractérisée par une granulométrie plus grossière et une plus grande richesse de minéraux ferromagnétiques. Dans ce cas précis, la susceptibilité peut donc être utilisé comme mesure directe de l'importance relative de la dynamique éolienne.

4 - Enfin, les premières tentatives d'analyse des loess lités par numérisation directe et analyse en niveaux de gris montrent des variations à très haute fréquence de la granulométrie au sein de ce faciès (millimétrique à centimétrique). Cette lamination, typique de la période 25-20 ka environ, est interprétée, d'après les données de Nussloch, comme la trace de tempêtes de poussière particulièrement intenses au Pléniglaciaire supérieur, capables de remanier des matériaux sableux à partir de la plaine alluviale du Rhin. 


\section{REMERCIEMENTS}

Les auteurs remercient vivement $M$. Löesher pour son aide et la chaleur de son accueil sur le terrain, ainsi que la compagnie Heidelberger Zement Company qui nous permet de travailler régulièrement et dans de bonnes conditions sur le site de Nussloch. (Contribution ISEM 2002-087).

\section{RÉFÉRENCES}

ANTOINE P., LAUTRIDOU J.P., SOMMÉ J., AUGUSTE P., AUFFRET J.P, BAIZE S., CLET-PELLERIN M., COUTARD J.P., DEWOLF Y., DUGUÚ O., JOLY F., LAIGNEL B, LAURENT M., LAVOLLE M., LEBRET P., LÉCOLLE F., LEFEBVRE D., LIMONDIN-LOZOUET N., MUNAUT A.V, OZOUF J.C., QUESNEL F et ROUSSEAU D.D. 1998 - Le Quaternaire de la France du Nord-Ouest : Limites et Corrélations. Quatemaire. 9 (3), 227-241, 1 pl. HT.

ANTOINE P., ROUSSEAU, D.D., LAUTRIDOU J.P., \& HATTÉ, C. 1999 - Last Interglacial-Glacial climatic cycle in loess-palaeosol successions of north-western France, Boreas, 28, 551-563.

ANTOINE P., ROUSSEAU D.D., ZÖLLER L., LANG A., MUNAUT, A.V., HATTE C. \& FONTUGNE M. 2001 - High resolution record of the last Interglacial-glacial cycle in the Nussloch loess-palaeosol sequences, Upper Rhine Area Germany. Quaternary International, 76-77, 211-229.

BÉGÉT J.E., STONE D.B. \& HAUWKINS D.B. 1990 - Paleoclimatic forcing of magnetic susceptibility variations in Alaska loess during the Late Quaternary. Geology, 18, 40-43.

BENTE B. \& LÖSHER M, 1987 - Sedimentologishe, pedologishe und stratigraphishe Unterschungen an Lössen südlich Heidelberg. Göttinger geogr. Abh., 84, 9-17.

BIBUS E. 1980 - Zur Relief-, Boden- und Sedimententwicklung am unteren Mittelrhein. Frankfurter geowiss. Arb. , D1, 296 pp.

CHLACHULA J., EVANS M.E \& RUTTER N.W. 1998 - A magnetic investigation of a Late Quaternary loess/palaeosol record in Siberia. Geophysical Internationa Journal, 132, 128-132.

De ANGELIS M., J.P. STEFFENSEN M.R., LEGRAND H.B., CLAUSEN H.B, and HAMMER C.U., 1997 - Primary aerosol (sea salt and soil dust) deposited in Greenland ice during the last climatic cycle: Comparison with east Antarctic records. Journal of Geophysical Research, 102, 26681-26698.

DERBYSHIRE E., MENG X. \& KEMP R. 1998 - Provenance, transport and characteristics of modem aeolian dust in western Gansu province, China, and interpretation of the Quatemary loess record. Journal of Arid Environments, 39, 497-516.

ELVERHØI A., ANDERSEN E.S., DOKKEN T., HEBBELN D., SPIELHAGEN R., SVENDSEN J.I., SøRFLATEN M., RøRNES A., HALD M., \& FORSBERG C.F. 1995 - The growth and decay of the Late Weichselian ice scheet in western Svalbard and adjacent areas based on provenance studies of marine sediments. Quaternary Research, 44, 303-316.

FRECHEN M. 1999 - Upper Pleistocene loess stratigraphy in Southern Germany. Quaternary Geochronology, 18, 243-269. GRIP members, 1993 - Climate instability during the last interglacial period recorded in the GRIP ice-core. Nature, 364, 203-207.

GROUSSET F. 2002 - Les changements abrupts du climat depuis 60000 ans. Quaternaire, 12, 203-211.

HAESAERTS P. 1985 - Les loess du Pléistocène Supérieur en Belgique ; comparaison avec les séquences de l'Europe Centrale. Bulletin de l'Association Française pour l'Étude du Quaternaire, 22, 105-115.

HAESAERTS P. \& Van VLIET.LANOË B. 1981 - Phénomènes périglaciaires et sols fossiles observés à Maisières-Canal, à Harmignies et à Rocourt. Biultyn. peryglacalny, 28, 291-324.

HAESAERTS P. JUVIGNÉ E., KUYL O, MÜCHER H. \& ROEBROEKS W. 1981 - Compte rendu de l'excursion du 13 Juin 1981, en Hesbaye et au Limbourg Néerlandais, consacrée à la chronostratigraphie des loess du Pléistocène supérieur. Annales de la Societé Géologique de Belgique, 104, 223-240.
HAESAERTS P., MESTDAGH H., GERMONPRÉ M., \& DAMBLON F. 19969 - Reasearch Network SC-004, General Report on the activities of the Network since 01/11/92. Institut Royal des Sciences Naturelles de Belgique. 55 pp.

HAESAERTS P., DAMBLON F., BACHNER, G., TRNKA, G. 1996b - Revised stratigraphy and chronology of the Willendorf II sequence, Lower Austria. Archaeologia Austriaca Verlag Franz Deuticke, Wien, 25-42.

hatTÉ C., FONTUGNE M., ROUSSEAU D.D., ANTOINE P., ZöLLER L. TISNERA-LABORDE N., and BENTALEB I. $1998-\delta{ }^{13} \mathrm{C}$ Variations of loess organic matter as a record of the vegetation response to climatic changes during the Weichselian. Geology, 26 (7), 583-586.

hatTt C., ANTOINE P., FONTUGNE M., ROUSSEAU D.D., TISNERA-LABORDE, N., and ZÖLLER, L. 1999 - New chronology and organic matter $\delta{ }^{13} \mathrm{C}$ paleoclimatic significance of Nußloch loess sequence (Rhine Valley, Germany). Quaternary International, 62, 85-91.

HATTE C., ANTOINE P., FONTUGNE M., LANG A., ROUSSEAU D.D., \& ZöLLER L. $2001-\delta^{13} \mathrm{C}$ of loess organic matter as a potential proxy for paleoprecipitation.Quatemary Research, 55, 33-38.

HELLER, F. \& EVANS, M.E. 1995 - Loess magnetism, Reviews of Geophysics, 33 (2), 211-240.

HUIJZER B. \& VANDENBERGHE J. 1998 - Climatic reconstruction of the Weichselian Pleniglacial in northwestern and central Europe. Journal of Quaternary Science, 13 (5), 391-417.

JUVIGNÉ E. \& SEMMEL A. 1981 - Un tuf volcanique semblable à l'Eltviller Tuff dans les loess de Hesbaye et du Limbourg nérlandais. Eiszeit. und Gegenwart, 31, 83-90

LAUTRIDOU J.P. et SOMMÉ J. 1974 - Les loess et les provinces climato-sédimentaires du Pléistocène supérieur dans le NordOuest de la France. Bulletin de l'Association Française pour l'Étude du Quaternaire, 11, 237-241.

LAUTRIDOU J.P. 1985 - Le cycle périglaciaire pléistocène en Europe du Nord-Ouest et plus particulierement en Normandie. Thèse es Sciences, Univ. Caen, Centre de Géomorphologie Caen, 908pp.

LÉGER M. 1990 - Loess landforms. Quaternary International, 7/8, 53-61.

MAHER B.A. \& TAYLOR R.M. 1988 - Formation of ultrafine-grained magnetite in soils. Nature, 336, 368-370.

MAHER B. and THOMPSON R. 1992 - Paleoclimatic significance of the mineral magnetic record of the Chinese Loess and Paleosols. Quaternary Research, 37, 155-170.

MOINE O., ROUSSEAU D.D. et ANTOINE P. 2002 - Mise en évidence d'événements climatiques rapides par les faunes de mollusques terrestres des loess weichselien de Nussloch (Allemagne). Quaternaire, 13 (3/4) (sous-presse).

NAWROCKI J., WOJCIK A., BOGUCKI A. 1996 - The magnetic susceptibility record in the Polish and western Ukrainian loesspalaeosol conditioned by palaeoclimate. Boreas, 25, 161-169.

PORTER S. \& AN Z. 1995 - Correlation between climate events in the North Atlantic and China during the Last Glaciation. Nature, $375,305-308$.

PORTER S.C. 2000 - High-resolution paleoclimatic information from Chinese eolian sediments based on grayscale intensity profiles, Quaternary Research, 53, 70-77.

ROUSSEAU D.D., SOUTARMIN N., GAUME L., ANTOINE P., LANG M., LAUTRIDOU J.P., SOMME, J., ZÖLLER L., LEMEUR I., MEYNARDIER i., FONTUGNE M. \& WINTLE A. 1994 - Histoire du Dernier cycle climatique enregistrée dans la séquence loessique d'Achenheim (Alsace, France), à partir de la susceptibilité magnétique. Comptes Rendus de l'Académie des Sciences, Paris, 319, Série II, 551-558.

ROUSSEAU D.D., ZÖLLER L. \& VALET J.P. 1998 - Late Pleistocene climatic variations at Achenheim, France, based on a magnetic susceptibility and TL chronology of loess. Quaternary Research, 49, 255-263. 
ROUSSEAU D.-D., ANTOINE P., HATTÉ C., LANG A., ZÖLLER L., FONTUGNE M., BEN OTHMAN D., LUCK J.-M. MOINE O., LABONNE M., BENTALEB I. \& JOLLY D., 2002 - Abrupt millenial climatic changes from Nussloch (Germany) Upper Weichselian eolian records during the Last Glaciation. Quaternary Science Reviews, 21, 1577-1582.

SABELBERG U. \& LÖSCHER M. 1978 - Neue Beobachtungen zur Würmlöss-Stratigraphie südlich Heidelberg. in Festschr. J. Fink, Wien, Hirt.

VANDENBERGHE J., AN Z., NUGTEREN G., HUAYU L. \& VAN HUISSTEDEN K. 1997 - New absolute time scale for the Quaternary climate in the Chinese loess region by grain-size analysis. Geology, 25 (1), 35-38.

VANDENBERGHE J., HUIJZER B., MÜCHER H. \& LAAN W. 1998 - Short climatic oscillations in a western European loess sequence (Kesselt,Belgium). Journal of Quaternary Science, 13, 471-485.

VAN VLIET-LANOË B. 1987 - Le rôle de la glace de ségrégation dans les formations superficielles de l'Europe du Nord-Ouest. Thèse de Doctorat d'Etat, Université Paris I, $864 \mathrm{pp}$.

XIAO J. PORTER S.C., AN Z., KUMAIH. \& YOSHIKAWA S. 1995 - Grain size of quartz as an indicator of winter monsoon strenght on the Loess Plateau of Central China during the Last $130000 y r .$, Quaternary Research, 43, 22-29.

ZHOU L.P., OLDFIELD F., WINTLE A.G., ROBINSON S.G. \& WANG J.T. 1990 - Partly pedogenic origin of magnetic variations in Chinese loess. Nature, 346, 737-739.

ZÖLLER L., STREME H.E. \& WAGNER G.A. 1988 - Thermolumineszenz-Datierung an Löss-Paläoboden-Sequenzen von Nieder-, Mittle-und Obberhein. Chemical Geollogy, Isot. Geosc. Sect., 73, 39-62.

ZÖLLER L. \& WAGNER G. 1990 - Thermoluminescence dating of loess-recent developments. Quaternary International, 7/8, 119-128. 